\title{
Dermatitis irritativa relacionada con la higiene de manos en profesionales de enfermería de una unidad hospitalaria del ISSEMyM
}

\author{
Reyna Miliar-de Jesús ${ }^{1}$ e Iván Renato ZúñIga-Carrasco ${ }^{2 *}$ \\ ${ }^{1}$ Centro Médico, Instituto de Seguridad Social del Estado de México y Municipios; ${ }^{2}$ Unidad de Medicina Familiar 223, Instituto Mexicano del Seguro Social, Lerma, \\ Méx., México
}

\begin{abstract}
Introduction: Hand dermatitis is a frequent condition within occupational skin diseases, with a prevalence of 80-88\%; it can be explained by the fact that hands are the most used «tools» of the body. Objective: To determine the prevalence of irritant dermatitis related to hand hygiene in the nursing staff at a social security hospital. Methods: An observational, descriptive, cross-sectional and prospective study was carried out. Study subjects were the nursing staff of the special shift; they were asked for their informed consent and the modified questionnaire of the World Health Organization (WHO) Observer Manual for hand hygiene was applied. Results: We included 72 participants, $87 \%$ female and 13\% male, with an age range of 20-54 years. The prevalence of irritant hand dermatitis was $41.6 \%$, being higher (38\%) in subjects with light-brown skin. Scales were seen in $13 \%$ as very severe damage on the ridges, and $5 \%$ presented cracks. Conclusions: The nursing staff of the special shift showed good adherence to the hand hygiene program. To strengthen this adherence, an inter-institutional study on tolerability to the guidelines established by the WHO is necessary so that the staff can decide which is the product that does not cause dermatitis. (Hosp Med Clin Manag. 2019;12:69-75)

Corresponding author: Iván Renato Zúñiga-Carrasco, ivan.zuniga@imss.gob.mx
\end{abstract}

Key words: Dermatitis. Occupational contact dermatitis. Nurse. Hand washing. Moisturizing creams. Skin.

\section{RESUMEN}

Introducción: La dermatitis de mano es frecuente dentro de las enfermedades cutáneas ocupacionales, con una prevalencia del 80-88\%, y puede explicarse por el hecho de que las manos son las «herramientas» corporales más utilizadas. Objetivo: Determinar la prevalencia de dermatitis irritativa relacionada con la higiene de manos en los profesionales de enfermería de una unidad hospitalaria. Métodos: Se realizó un estudio observacional, descriptivo, transversal y prospectivo. El universo de trabajo fue el personal de enfermería del turno especial, a quienes se les solicitó su consentimiento informado y se les aplicó el cuestionario modificado del Manual para Observadores de la Organización Mundial de la Salud para el mejoramiento de la higiene de manos. Resultados: Se incluyeron

\section{Correspondencia:}

*Iván Renato Zúñiga-Carrasco

E-mail: ivan.zuniga @imss.gob.mx
Date of reception: 07-12-2018

Date of acceptance: 17-05-2019

DOI: 10.24875/HMCM.19000176 
72 participantes, $87 \%$ del sexo femenino y $13 \%$ del masculino; el rango de edad fue de $20-54$ años. La prevalencia de dermatitis irritativa fue del $41.6 \%$, teniendo mayor predisposición (38\%) las personas de piel morena clara. El 13\% presentó escamas como daño muy severo en los rebordes y el 5\% fisuras. Conclusión: La dermatitis de mano es una patología que afecta al personal de enfermería debido a la exposición a agentes irritantes como soluciones antisépticas, jabonosas, material de látex y la frecuencia en la higiene de manos.

Palabras clave: Dermatitis. Dermatitis ocupacional. Enfermería. Lavado de manos. Cremas humectantes. Piel.

\section{INTRODUCCIÓN}

La dermatitis de mano (DM) representa un problema de salud en los países industrializados; suele ser una manifestación de tipo irritativa por contacto o alérgica. Alergia y dermatitis atópica son endógenas. Podemos agregar que la influencia ambiental representa un importante factor de riesgo exógeno en el desarrollo de este padecimiento. La exposición ocupacional es particularmente relevante por diversos alergenos de contacto potenciales, entre los que destacan el agua y el látex, los cuales se encuentran y utilizan durante toda la jornada laboral de los profesionales de la salud; la frecuencia en el lavado de manos es también conocida por ser un factor de riesgo importante. Por esta razón, el profesional de salud es regularmente afectado por la DM hasta en un $70 \%$, reportando al menos una vez al año esta condición. El personal más afectado es el de enfermería, quienes tienen un riesgo particularmente alto.

\section{FACTORES DE RIESGO PARA DERMATITIS DE MANO}

Entre los factores de riesgo identificados para DM se encuentra una historia previa de enfermedad alérgica, y el riesgo de DM aumenta en proporción a la frecuencia del lavado de manos entre el personal de salud del hospital incluyendo el de enfermería. Otro factor de riesgo que se ha observado es el lavado de manos más de 15 veces por turno de trabajo, el cual aumenta el riesgo de DM. Asimismo, laborar en áreas quirúrgicas, el consumo de alcohol y el uso de guantes de látex aumentan el riesgo. La influencia estacional significativamente está correlacionada con la DM. La piel con atopia se irrita con mayor facilidad que la de las personas no atópicas, y generalmente tienen frecuentes recaídas. Los síntomas agudos de la DM suelen ser más severos entre los atópicos. Los mecanismos anteriormente comentados contribuyen en gran medida a explicar la naturaleza de la DM entre el personal de enfermería y otro personal de salud. Se ha reportado que los productos para la higiene y lavado de manos son la causa más común para DM dentro de un hospitall $^{1,2}$. La piel seca es un factor de riesgo, particularmente en combinación con dermatitis atópica. Haber padecido eczema infantil es otro factor de riesgo para la $\mathrm{DM}^{3}$.

\section{OCUPACIÓN Y DERMATITIS DE CONTACTO OCUPACIONAL}

Los grupos ocupacionales que tienen la más alta prevalencia de dermatitis por contacto con sustancias en el trabajo son: médicos, odontólogos, enfermeras, farmacéuticos, dietistas, terapeutas, tecnólogos, técnicos y asistentes ${ }^{4,5}$.

Entre los trabajadores de la salud, el personal de enfermería tiene una prevalencia puntual estimada del 12-30\% para desarrollar DM. Casi el $60 \%$ de los trabajadores de la salud reportan tener eczema durante el primer año después de la notificación de la enfermedad ${ }^{6}$. La DM no solo afecta la calidad de vida, sino que en casos prolongados y frecuentes recaídas puede llevar a la pérdida del empleo. Una vez que un individuo ha desarrollado $\mathrm{DM}$, el pronóstico es pobre. Una tercera parte de las personas que tienen DM necesita atención médica y tratamiento, el $5 \%$ experimenta periodos prolongados de la enfermedad, así como ausentismo, pérdida o cambio de trabajo, y en casos más severos pensión por enfermedad. Las personas afectadas también pueden experimentar consecuencias psicosociales, tales como alteraciones del sueño e interferencia con actividades de esparcimiento ${ }^{7}$. La dermatitis de contacto ocupacional (DCO) es frecuente entre los trabajadores de la salud, con una prevalencia de hasta el 30\%. La DCO en el personal de la salud surge por el contacto frecuente con 
los productos para la higiene y lavado de manos, uso de soluciones desinfectantes para superficies, instrumental, manejo de medicamentos y uso de guantes. Entre los alergenos más frecuentemente reportados en enfermeras con DCO se pueden encontrar varios preparados tales como: yodopovidona $10 \%$, glutaraldehído $0.3 \%$, propilenglicol 20\%, 1,3-difenil guanidina $1 \%$ y octilgalato $0.3 \%^{8}$. La DCO tiene una presentación clínica con prurito, enrojecimiento, placas, pápulas, vesículas y fisuras que se desarrollan después del contacto con un irritante o químico alergénico. A menudo se describe como eczema clásico con purito. La DM en el personal de salud es del $95 \%$ y afecta principalmente a las mujeres; la mayoría de las veces comienza en los dedos?

En la práctica, cuando un profesional de la salud no es alérgico al caucho, el uso de guantes provoca que la DM existente empeore debido a la oclusión, sudoración y maceración ${ }^{10}$. Otro factor precipitante es el contacto por el uso de anillos, principalmente aquellos niquelados, ya que las reacciones alérgicas a menudo ocurren cuando jabones, cremas cosméticas y talco de los guantes se acumulan debajo de la joyería. Algunos metales, especialmente aleaciones de cobre y plata, pueden servir como irritantes primarios en presencia de una concentración adecuada de sodio. En general, el eczema de manos comienza bajo un anillo. La erupción puede extenderse desde el dedo hacia la mano u otros dedos y puede conducir a infección secundaria o sensibilización al látex ${ }^{10}$.

Los productos hidratantes de la piel son de uso común entre el personal de salud. Existen políticas y medidas de seguridad del hospital que los prohíben; sin embargo, el personal de enfermería usa una gran variedad de productos para hidratarse las manos, lo que aumenta el riesgo de contaminación e incompatibilidad con los productos que se utilizan para la higiene y lavado de manos ${ }^{11}$. Aunque se encuentra restringido el uso de cremas hidratantes, se ha demostrado que ayudan a disminuir los síntomas de DM y el engrosamiento de la piel, por lo que se permite que se utilicen al final de la jornada laboral. Las cremas humectantes evitan el resecamiento y pérdida de lípidos en la piel, ya que tienen propiedades de barrera, ayudando a restaurar la capacidad de retención de agua y aumentar el ancho de los corneocitos. Como recomendación se deben utilizar cuatro veces al día ${ }^{12-14}$. Otras ocupaciones que tienen mayor riesgo de desarrollar DCO son estilistas, y trabajadores en las áreas de la construcción, ingeniería y electrónica ${ }^{15}$.
Por otra parte, la exposición de las manos u otras partes del cuerpo a ambientes fríos y vientos secos causa cambios e irritación en la piel tales como sequedad, fisuras, agrietamiento de la piel, descamación y escoriación. Las condiciones climáticas pueden ser un factor de riesgo para provocar alteraciones visibles de la superficie de la piel ${ }^{16-18}$. Es importante destacar la magnitud de los problemas de la piel en los trabajadores de la salud, resaltando que no se conocen del todo, así como los factores asociados con el daño, los cuales no han sido evaluados sistemáticamente ${ }^{19,20}$. En un estudio sobre reacciones cutáneas adversas a la solución base de alcohol gel, se concluyó que es importante para los profesionales de la salud ser informados sobre el reconocimiento y manejo de la dermatitis de contacto ${ }^{21}$.

El agua ha sido identificada como un irritante de la piel, principalmente cuando contiene cloro. La exposición repetida al agua causa dilución de factores humectantes naturales en el estrato córneo. La propiedad de retención de agua del estrato córneo es proporcionada en parte por lípidos intercelulares, los cuales se encuentran de forma abundante en las secreciones de glándulas sebáceas. Cuando el profesional de la salud se realiza lavado de manos de forma repetida ocurre esa pérdida de lípidos del estrato córneo, resecando la piel y al contacto con el jabón provoca irritación en las manos que favorece la $\mathrm{DM}^{22}$. Llevamos a cabo un estudio para determinar la prevalencia de dermatitis irritativa relacionada con la higiene de manos en los profesionales de enfermería en una unidad hospitalaria.

\section{MÉTODOS}

Previa autorización del Comité Institucional de Investigación en Salud y Ética en Investigación, se realizó un estudio observacional, descriptivo, transversal y prospectivo. El universo de trabajo fueron 72 trabajadores de enfermería de diversos servicios, del turno especial de la Unidad Médica Instituto de Seguridad Social del Estado de México y Municipios (ISSEMyM). Criterios de selección: a) personal de enfermería que laborara en el turno especial; b) que perteneciera a diferentes categorías: auxiliares, generales, especialistas, jefes de piso, estudiantes de enfermería, pasantes de enfermería o suplentes; c) que deseara colaborar con el trabajo de investigación; d) que laborara en los Servicios de terapia intensiva, urgencias, hospitalización cirugía, hospitalización medicina interna, quirófano, hemodiálisis, diálisis, 


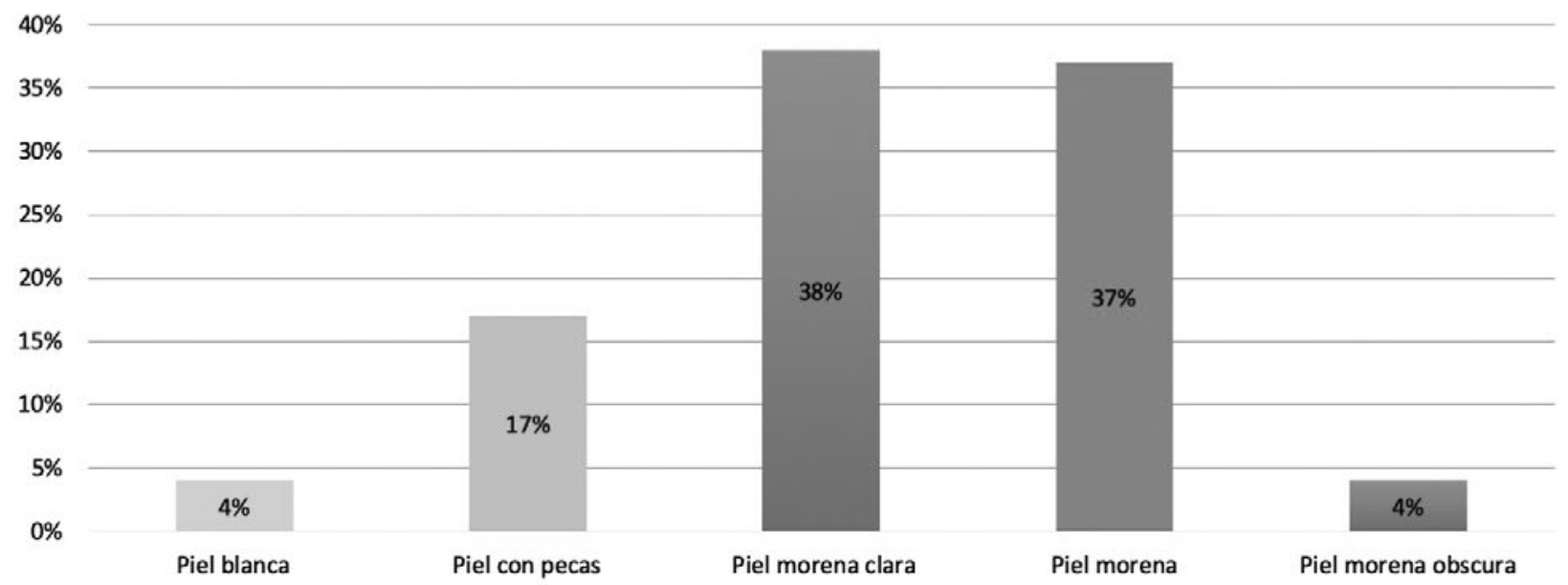

Figura 1. Tipo de piel con predisposición a dermatitis.

trasplantes o central de esterilización y equipos. Los criterios de exclusión fueron: a) personal que no laboró el día del estudio; b) con diagnóstico de atopia; c) personal médico adscrito, médicos internos de pregrado, médicos residentes y estudiantes médicos. A los participantes se les solicitó su consentimiento informado y se les aplicó el cuestionario modificado del «Manual para Observadores, Estrategia Multimodal para el Mejoramiento de la Higiene de las Manos de la Organización Mundial de la Salud" $(\mathrm{OMS})^{23}$, siendo primavera la estación del año en la cual se aplicó el cuestionario.

\section{RESULTADOS}

Se incluyeron 72 participantes, $87 \%$ del sexo femenino y $13 \%$ del sexo masculino, con rango de edad de 20 a 54 años. Con relación al color de la piel del personal de enfermería, fue como sigue: piel blanca, $4 \%$; piel con pecas, $17 \%$; piel morena clara, $38 \%$; piel morena, $37 \%$; piel morena oscura, $4 \%$. Se pudo observar que, en el caso de las participantes con piel morena clara, el 38\% tenía más predisposición a tener DM, la piel morena un $37 \%$, la piel con pecas un $17 \%$, la piel morena oscura un $4 \%$ y la piel blanca un $4 \%$; el $11 \%$ restante no presentó ningún tipo de DM (Fig. 1).

\section{Patologías alérgicas}

El $58 \%$ de los participantes comentó que las actividades no relacionadas con el trabajo probablemente pueden afectar la piel. Se les preguntó si utilizaban normalmente una crema protectora para las manos, respondiendo: con mayor frecuencia, 38\%; varias veces al día, 33\%; una vez al día, $8 \%$; a veces, $8 \%$; y en raras ocasiones, $13 \%$. En cuanto a la presencia de dermatitis irritativa, los participantes respondieron: nunca, $13 \%$; a veces, $80 \%$; siempre, $7 \%$; no sufre dermatitis atópica, $88 \%$; $92 \%$ no sufre rinitis o conjuntivitis. Ningún participante padecía asma.

\section{Uso de jabón}

El tiempo que llevaban utilizando el jabón para la higiene de las manos en el trabajo fue: < 1 año, 14\%; $1-5$ años, 31\%; y $>5$ años, 55\%. El producto institucional utilizado se evaluó de la siguiente manera en el rubro irritación: muy irritante, $63 \%$; moderadamente irritante, $20 \%$; y no irritante, $17 \%$. Sobre el efecto desecante del producto utilizado: muy importante, $67 \%$; moderado, $16 \%$; y nada desecante, $17 \%$. La evaluación global del producto fue: insatisfecho, 38\%; moderadamente satisfecho, 30\%; y muy satisfecho, $32 \%$. En el rubro sobre qué producto prefería: producto institucional, $36 \%$; producto propio, 59\%; y ninguno, $5 \%$. Al preguntárseles si el producto mejoraba el cumplimiento de la higiene de manos respondieron: rotundamente sí, $33 \%$; en absoluto, $59 \%$; y de ninguna manera, $8 \%$.

Las sustancias que pueden contribuir a DM se desglosan en la figura 2, siendo el detergente y el cloro las sustancias que más utilizan en sus labores domésticas.

\section{Evaluación del producto}

Al evaluar el producto con respecto a las condiciones de la piel se vio que tenía un aspecto anormal 54\%, moderado 


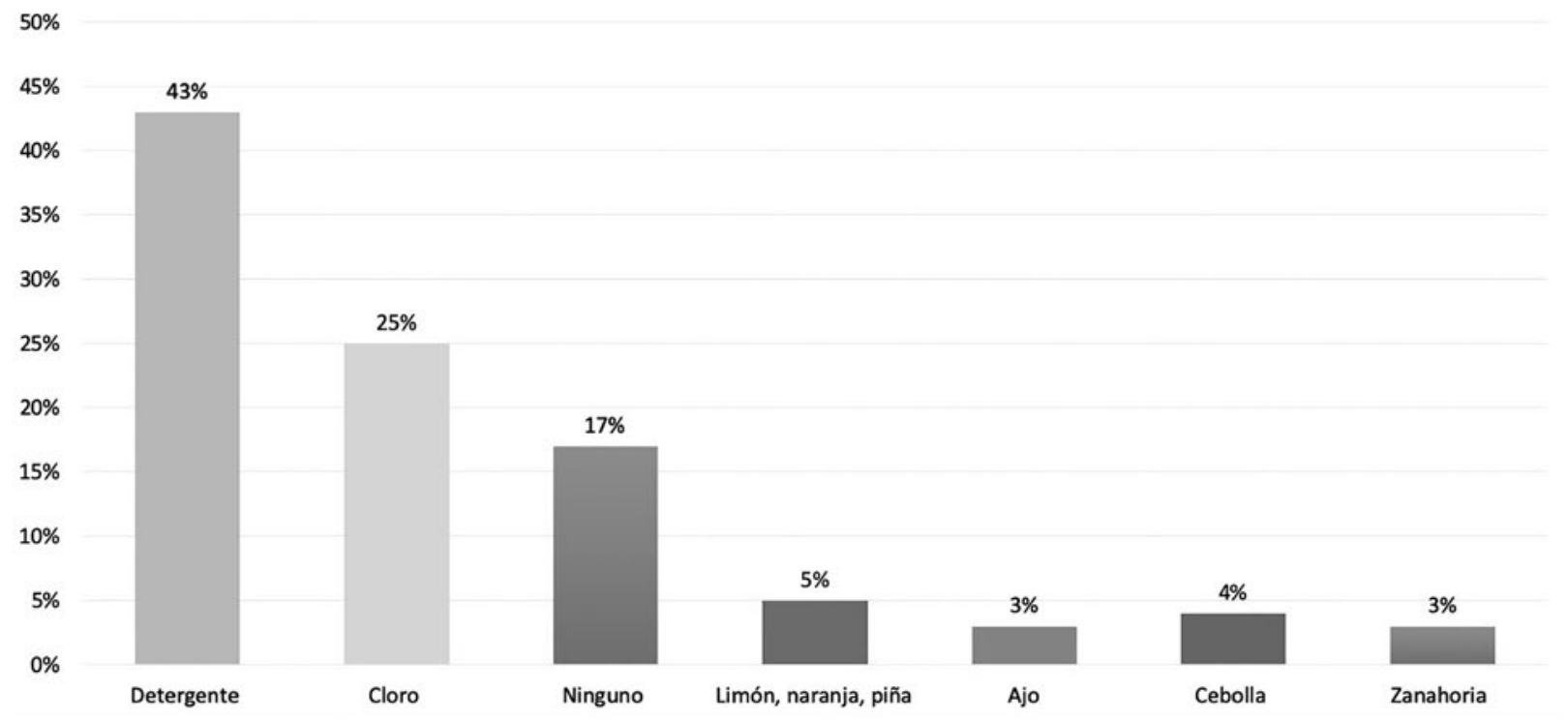

Figura 2. Sustancias que pueden contribuir a la dermatitis.

Tabla 1. Inspección visual de la piel al momento de aplicar el cuestionario

\begin{tabular}{|c|c|c|c|c|c|}
\hline Escala observador & Sin dano & Leve & Moderada & Severa & Muy severa \\
\hline Enrojecimiento & & Leve $83 \%$ & Moderado $17 \%$ & & \\
\hline Escamas & Sin escamas $67 \mathrm{~A}$ & Muy leves $21 \%$ & Moderadas $12 \%$ & & $\begin{array}{l}\text { Separation pronunciada de } \\
\text { los rebordes } 13 \%\end{array}$ \\
\hline Fisuras & Sin fisuras $50 \%$ & Muy finas $30 \%$ & Grandes $15 \%$ & & Grietas diseminadas $5 \%$ \\
\hline Piel seca & & Leve $49 \%$ & Moderada $51 \%$ & & \\
\hline Piel muy seca & & Leve $70 \%$ & Moderada 25\% & Severa $5 \%$ & \\
\hline Piel agrietada & & Lees 70', & Moderada 25\% & Severa $5 \%$ & \\
\hline
\end{tabular}

$25 \%$, normal 21\%; la integridad fue anormal en 59\%, moderada 25\%, normal 16\%; hidratación anormal 71\%, moderada 20\%, normal 9\%; sensación anormal 70\%; moderada $22 \%$; normal $8 \%$. Así evaluaron la integridad de la piel de las manos: muy alterada, 46\%; moderadamente alterada, 17\%; perfecta, 37\%. Se realizó una inspección visual de la piel al momento de aplicar el cuestionario (Tabla 1, Figs. 3-5)

\section{Discusión}

En nuestro estudio se pudo corroborar lo que algunos autores reportaron con respecto a la presencia de DM por el lavado habitual de manos ${ }^{3,4,6}$ : el personal de enfermería es el personal de salud que más se apega al Programa Institucional de Higiene de Manos, ya que el contacto con el paciente y su entorno es frecuente durante toda la jornada laboral; por ello, es la ocupación con una alta prevalencia de dermatitis. Por ejemplo, en este estudio los participantes reportaron lavarse las manos 30 a 35 veces durante el turno de trabajo.

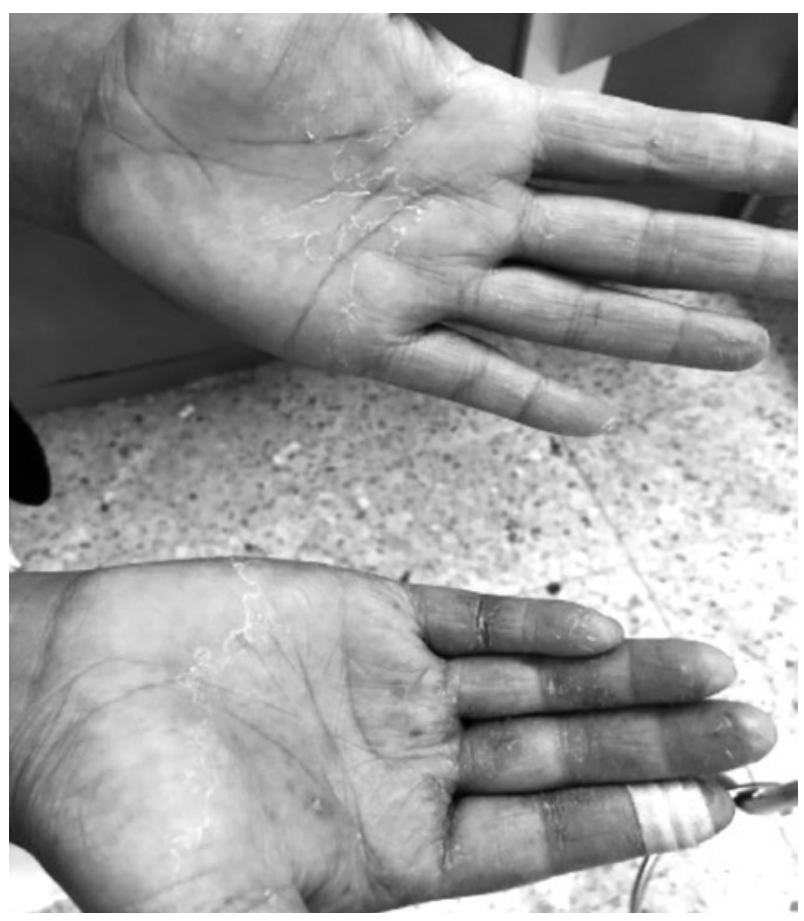

Figura 3. Piel agrietada severa. 


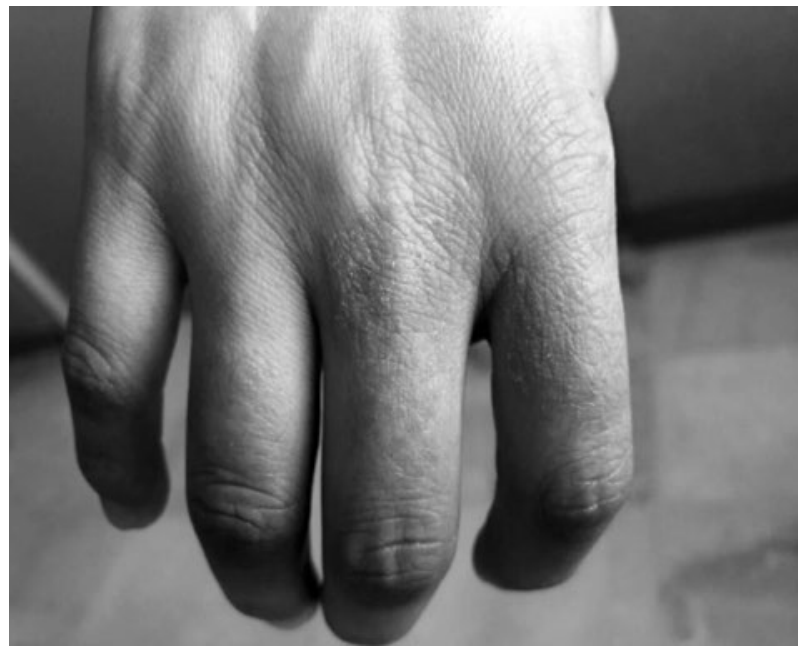

Figura 4. Piel seca moderada.

Como se puede observar en las imágenes de manos del personal entrevistado, presentan DM; los casos más severos, con varios meses de evolución, concuerdan con lo que nos mencionan en sus reportes Madan, et al. y Cimiotti, et al. ${ }^{7,9}$, siendo factores de riesgo para el desarrollo de DM el uso de guantes de látex y el de diversas sustancias durante la jornada del personal de enfermería, como lo mencionan Furay, et al. ${ }^{10}$ y Larson, et al. ${ }^{11}$.

Se observó que la DM por el lavado de las manos es más frecuente de lo que se puede apreciar. Esto nos conduce a un desapego del programa institucional de higiene de manos por la utilización complementaria de productos que no llegan a ser compatibles con los que otorga la institución, como se comenta en el reporte de Larson, et al. ${ }^{11}$.

La OMS en su «Manual para Observadores. Estrategia multimodal para el mejoramiento de la higiene de las manos. Alianza mundial para la seguridad del paciente. Primer Reto Global para la seguridad del paciente. Atención limpia, atención segura» ${ }^{23}$, nos dice de manera puntual: «La higiene de las manos puede practicarse limpiando las manos con un desinfectante alcohólico o lavándolas con agua y jabón»; lo anterior es porque el personal tiene la creencia de que la «higiene» se lleva a cabo solo cuando se usa una solución base alcohol gel. La eficacia del desinfectante para las manos depende de la calidad y cantidad del producto utilizado, así como del tiempo empleado en la higiene de manos. Estos parámetros también se aplican al lavado de las manos con agua y jabón. Según recomendaciones de

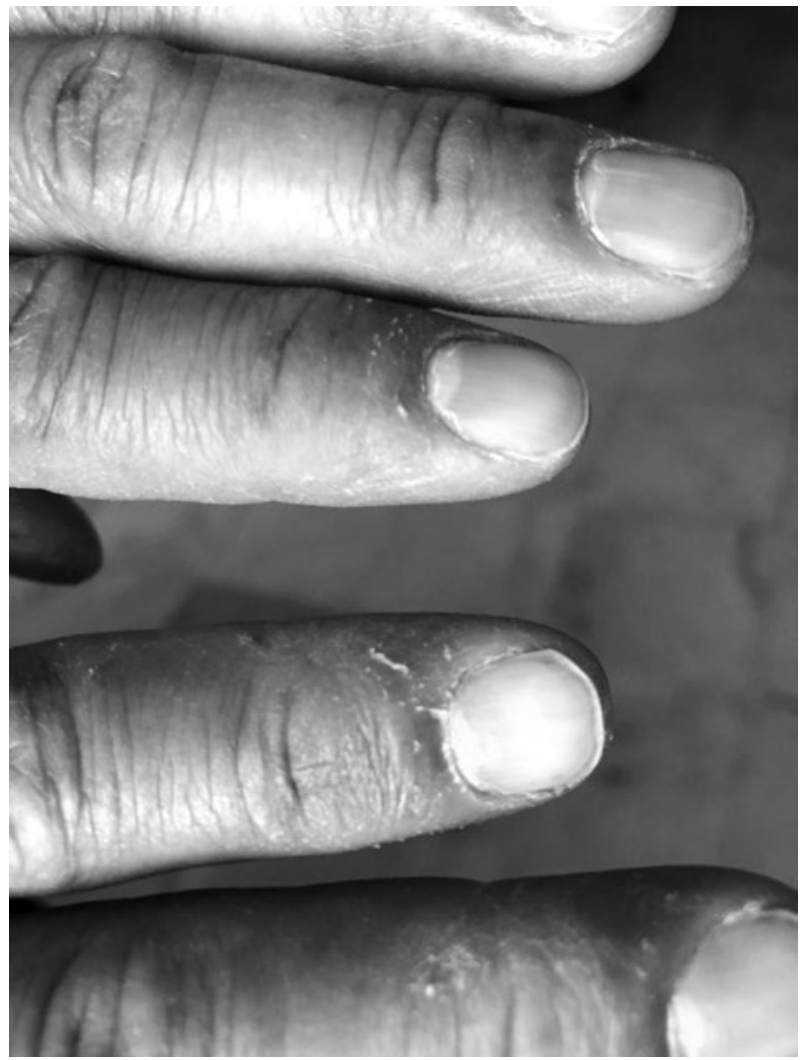

Figura 5. Piel seca moderada.

la OMS, uno de los factores que mejoran la aceptación de los productos de higiene de manos es la posibilidad de que los usuarios, en este caso el personal de enfermería, el cual como comentamos anteriormente tiene mayor apego a la higiene de manos, intervengan en su elección. Junto con la eficacia comprobada como antiséptico, la tolerabilidad de la piel es uno de los criterios principales de la selección de productos; un producto cuyo uso sea agradable y que carezca de efectos nocivos para las manos es un recurso importante para promover la práctica de la higiene de manos. Hay varias maneras de garantizar la aceptabilidad de un producto. El método más sencillo es la realización de un estudio de tolerabilidad de un producto que está en uso o que se prevé introducir. Un método diferente y más complejo permite comparar la tolerabilidad y la aceptabilidad de diferentes productos. Este método puede ser particularmente útil cuando deba elegirse entre diferentes productos, como nos recalcan Tukuda, et al. en su reporte ${ }^{21}$. Es importante la participación de personal de salud ocupacional, de dermatología y epidemiología, quienes deben estar al tanto de los casos de dermatitis provocados por la higiene de manos. Los primeros y los segundos, con el fin de determinar la 
severidad de las lesiones tomando acciones y un manejo oportuno. Epidemiología debe realizar una evaluación mensual del apego a la higiene de manos y valorar las causas del desapego a dicho proceso, tomando aspectos tan relevantes como son los factores de riesgo que se vuelven áreas de oportunidad para el Programa Institucional de Higiene de Manos.

\section{AGRADECIMIENTOS}

Al personal de enfermería del turno especial del Centro Médico ISSEMyM, cuya colaboración fue primordial para llevar a cabo este estudio.

\section{BIBLIOGRAFÍA}

1. Smith D, Ohmura K, Yamagata Z. Prevalence and correlates of hand dermatitis among nurses in a Japanese teaching hospital. J Epidemiol. 2003;13(3):157-61.

2. Smith $\mathrm{D}$, Wei N, Kang L, Wang R. Work environment and hand dermatitis among nurses in a Chinese teaching hospital. Environ Health Prevent Med. 2004; 9:181-4.

3. Smit H, van Rijssen A, Vandenbroucke J, Coenraads P. Susceptibility to and incidence of hand dermatitis in a cohort of apprentice hairdressers and nurses. Scand J Work Environ Health. 1994;20(2):113-21.

4. Behrens V, Seligman P, Caweron L, Mathias T, Fine L. The prevalence of back pain, hand discomfort and dermatitis in the US working population. Am J Public Health. 1994;84(11):1780-5.

5. Chen J, Gomez P, Kudla I, DeKoven J, Holness L, Skotnicki S. Return to work for nurses with hand dermatitis. Dermatitis. 2016;27(5):308-12.

6. Soltanipoor M, Kezic S, Sluiter J, Rustemeyer T. The effectiveness of a skin care program for the prevention of contact dermatitis in health care workers (the Healthy Hands Project): study protocol for a cluster randomized controlled trial. Trials. 2017;18:92.

7. Madan I, Parsons V, Cookson B, English J, Lavender T, McCrone P, et al. A behavioural change package to prevent hand dermatitis in nurses working in the national health service (the SCIN trial): Study protocol for a cluster randomised controlled trial. Trials. 2016;17:145.
8. Molin S, Bauer A, Schnuch A, Geier J. Occupational contact allergy in nurses: Results from the Information Network of Departments of Dermatology 2003-2012. Contact Dermatitis. 2014;72:164-71.

9. Cimiotti J, Marmur E, Nesin M, Hamlin P, Larson E. Adverse reactions associated with an alcohol-based hand antiseptic among nurses in a neonatal intensive care unit. Am J Infect Control. 2003;31:43-8.

10. Fay MF. Hand dermatitis: The role of gloves. AORN J. 1991;54(3):451-67.

11. Larson E, Norton C, Pyrek J, Sparks S, Cagatay E, Bartkus J. Changes in bacterial flora associated with skin damage on hands of health care personnel. Am J Infect Control. 1998;26:513-21.

12. Loden M. Barrier recovery and influence of irritant stimuli in skin treated with a moisturizing cream. Contact Dermatitis. 1997;36:256-60.

13. McCormick R, Buchman T, Maki D. A double-blind, randomized trial of scheduled use of a novel barrier cream and an oil-containing lotion for protecting the hands of healthcare workers. Am J Infect Control. 1998;26:468.

14. Larson E, Silberger M, Jakob K, Whittier S, Lai L, Della Latta P, et al. Assessment of alternative hand-hygiene regimens to improve skin health among neonatal intensive care unit nurses. Heart Lung. 2000;29:136-42.

15. Ramírez C, Jacob SE. Dermatitis de manos. Actas Dermo Sifiliográficas. 2006;97(6):363-73

16. Uter W, Gefeller 0 , Schwanitz $H$. An epidemiological study of the influence of season (cold and dry air) on the occurrence of irritant skin changes of the hands. Brit J Dermatol. 1998;138:266-72.

17. Parish WE. Chemical irritation and predisposing environmental stress (cold wind and hard water) En: Marks R, Plewig G, editores. The environmental threat to the skin. CRC Press; 1992.

18. Middleton JD, Allen BM. The influence of temperature and humidity on stratum corneum and its relation to skin chapping. J Soc Cosmet Chem. 1973; 24:239-43.

19. Larson E, Friedman C, Cohran J, Treston-Aurand J, Green S. Prevalence and correlates of skin damage on the hands of nurses. Heart Lung. 1997:26(5):404-12.

20. Dermatitis de manos en trabajadores de la salud. Programa de Evaluación e Investigación de Seguridad y Salud para la Prevención (SHARP) [Internet]. EE.UU.: Departamento de Trabajo e Industrias de Washington; 2001 [Consultado el 15/04/2018]. Disponible en: https://translate.google.com. $\mathrm{mx} /$ translate?hl=es-419\&sl=en\&u=http://www.Ini.wa.gov/Safety/Research/ Dermatitis/files/derm_hcw.pdf\&prev=search

21. Tukuda J, Kobayash $\mathrm{H}$. Effectiveness and problem of alcohol-based hand rub on hand hygiene. J Healthcare Associated Infect. 2009;2:7-12.

22. Larson E, Girard R, Pessoa-Silva C, Boyce J, Donaldson L, Pittet D. Skin reactions related to hand hygiene and selection of hand hygiene products. Am J Infect Control. 2006:34(10):627-35

23. Organización Mundial para la Salud. Manual para observadores. Guía de Implementación de la estrategia multimodal de mejoramiento de la higiene de las manos de la Organización Mundial de la Salud. Alianza mundial para la seguridad del paciente. Avaible at: https://www.paho.org/ cor/index.php option=com_docman\&view=download\&alias=235-manualpara-observadores-alianza-mundial-para-la-seguridad-del-paciente\&category_slug=calidad-y-seguridad-del-paciente\&Itemid=222 Consultado 1 de julio 2019. 